\title{
Práticas integrativas e complementares na Atenção Primária à Saúde: relato de experiência
}

\section{Integrative and complementary practices in Primary Health Care: experience report Prácticas integrativas y complementarias de la Atención Primaria de Salud: relato de experiencia}

\author{
Ivana Santos Ferraz iD \\ Universidade Estadual do Sudoeste da Bahia - Jequié - (BA) - Brasil
}

Sérgio Donha Yarid iD

Universidade Estadual do Sudoeste da Bahia - Jequié - (BA) - Brasil

\author{
Alba Benemérita Alves Vilela in \\ Universidade Estadual do Sudoeste da Bahia - Jequié - (BA) - Brasil \\ Universidade do Estado do Rio de Janeiro - Rio de Janeiro (RJ) - Brasil \\ Eduardo Nagib Boery \\ Universidade Estadual do Sudoeste da Bahia - Jequié - (BA) - Brasil \\ Ismar Eduardo Martins Filho \\ Universidade Estadual do Sudoeste da Bahia - Jequié - (BA) - Brasil
}

\section{RESUMO}

Objetivo: Relatar a vivência de mestrandos acerca do emprego das práticas integrativas e complementares como instrumento de construção de uma atenção integral à saúde em uma Unidade Básica de Saúde por profissionais de saúde do serviço. Síntese dos dados: Trata-se de um relato de experiência de cunho descritivo, o qual foi efetivado mediante a prática de campo de um grupo de mestrandos de um programa de pós-graduação. A experiência ocorreu em uma Unidade de Saúde do interior da Bahia, Brasil, em 2018. Na oportunidade, ofertaram-se vivências com as práticas integrativas e complementares para os mestrandos, tais como thetahealing, hipnose, quiropraxia e termalismo. O uso dessas práticas permitiu expandir o olhar acerca do cuidado em saúde pessoal e comunitário. As vivências serviram para comprovar os benefícios e aperfeiçoar o conhecimento para as atividades no ensino das práticas integrativas e complementares, sendo, de fato, uma experiência lisonjeira e importante, na medida em que forneceu conhecimento na prática, antes só vista na teoria. Conclusão: Observou-se como elemento fundamental para a execução das diferentes profissões da saúde a experiência de prática de campo com as terapias integrativas, sobretudo para os mestrandos e futuros docentes. Desse modo, se faz necessário que o conhecimento adquirido com essa experiência seja replicado em outros ambientes de saúde.

Descritores: Terapias Complementares; Saúde Pública; Atenção Primária à Saúde.

\section{ABSTRACT}

Objective: To report the experience of master's students about the use of Integrative and Complementary Practices as an instrument for building comprehensive health care in a Basic Health Unit by health professionals in the service. Data synthesis: It is a report of an experience of a descriptive nature, which was carried out through the field practice of a group of master's students in the Postgraduate Program in Nursing and Health at the State University of Southwest Bahia, Brazil. The experience took place at a Health Unit in the interior of Bahis, from November 21 to 25, 2018. At the time, students were offered some experiences with Integrative and Complementary Practices, such as Thetahealing, Hypnosis, Chiropractic and Thermalism. The use of these aforementioned practices allowed us to expand our view of personal and community health care. In addition to well-being and stress relief, the experiences served to prove the benefits, improve knowledge for activities in teaching about Integrative and Complementary Practices, being in fact a flattering and important experience, as it provided knowledge in practice before only seen in theory. Conclusion: It was observed as a fundamental element for the execution of the different health professions, the experience of field practice with integrative therapies, especially for master students and future teachers. Thus, it is necessary

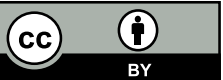


that the knowledge acquired from this experience be replicated in other health environments, spreading the knowledge about public policies with users, professionals and the academic community.

Descriptors: Complementary Therapies; Public Health; Primary Health Care.

\section{RESUMEN}

Objetivo: Relatar la vivencia de alumnos de maestría sobre la aplicación de prácticas integrativas y complementarias de profesionales sanitarios para la construcción de la atención integral de salud de una Unidad Básica de Salud. Síntesis de datos: Se trata de un relato de experiencia de carácter descriptivo de una práctica de campo de un grupo de alumnos de maestría de un programa de posgrado. La experiencia se dio en una Unidad de Salud del interior de Bahía, Brasil, en 2018. En la oportunidad se ha realizado vivencias con las prácticas integrativas y complementarias para los alumnos de maestría tales como el thetahealing, la hipnosis, la quiropraxia y el termalismo. El uso de esas prácticas ha permitido la ampliación de la mirada sobre el cuidado de salud personal y comunitaria. Las vivencias permitieron la comprobación de los beneficios y el perfeccionamiento del conocimiento para las actividades de enseñanza de las prácticas integrativas y complementarias, siendo, de hecho, una experiencia lisonjera e importante en la medida de que ha permitido el conocimiento práctico que solamente era visto en la teoría. Conclusión: Se observó la experiencia de la práctica de campo con las terapias integrativas como un elemento fundamental para la ejecución de las distintas profesiones de salud sobre todo para los alumnos de maestría y los futuros docentes. De esa manera, se hace necesario que el conocimiento adquirido con esa experiencia sea duplicado en otros ambientes de salud.

Descriptores: Terapias Complementarias; Salud Pública; Atención Primaria de Salud.

\section{INTRODUÇÃO}

As práticas integrativas e complementares (PIC) têm como intuito o cuidado integral ao paciente, levando em consideração o corpo e a mente desses indivíduos ${ }^{(1)}$, ressaltando-se que não substituem o tratamento tradicional. São um adicional, um complemento no tratamento, sendo indicadas por profissionais específicos conforme as necessidades de cada caso.

A Organização Mundial da Saúde (OMS), há pelo menos cinquenta anos, tem incentivado que sejam consideradas como recursos de cuidado pelos sistemas nacionais de saúde as medicinas tradicionais complementares e integrativas (MTCl), conhecidas como práticas e saberes em saúde tradicionais ou diversos da biomedicina. Nesse sentido, a Declaração de Alma Ata ampliou a Atenção Primária à Saúde (APS) como elemento estratégico de organização de sistemas públicos universais de saúde, com o objetivo de tornar universal o cuidado clínico sanitário às populações ${ }^{(2)}$.

Vale mencionar que outro aspecto primordial referente às PIC, além de outros benefícios, reside no fato delas gerarem minimização de gastos em virtude do baixo custo de sua realização, unindo competência terapêutica maior ou igual aos outros tipos de tratamento, com valor econômico acessível, fator importante para o sistema público de saúde( ${ }^{(3)}$.

Dessa forma, foram normatizadas várias PIC, em virtude da Política Nacional de Práticas Integrativas e Complementares (PNPIC). Como exemplo, podem ser citadas: a acupuntura, a homeopatia, a fitoterapia e o termalismo social/crenoterapia ${ }^{(4)}$, além da prática da medicina antroposófica ${ }^{(5)}$. Acrescentaram-se 14 novas práticas de saúde à PNPIC no ano de 2017, quais sejam: arteterapia, ayurveda, biodança, dança circular, meditação, musicoterapia, naturopatia, osteopatia, quiropraxia, reflexoterapia, reiki, shantala, terapia comunitária integrativa (TCl) e yoga ${ }^{(6)}$. Um ano após, em $2018^{(7)}$, adicionaram-se outras práticas, tais como: a aromaterapia, apiterapia, bioenergética, constelação familiar, cromoterapia, geoterapia, hipnoterapia, imposição de mãos, ozonioterapia e terapia de florais.

Em 2016, havia PIC em 9.470 estabelecimentos de saúde difundidos em 56\% (3.097) dos municípios brasileiros, e na APS, em $54 \%$ deles. Estava concentrada nas capitais, especialmente nas regiões Sul e Nordeste. O maior número desses estabelecimentos era público e de administração municipal (APS). Um mesmo estabelecimento de saúde pode ofertar mais de uma modalidade de PIC, homeopatia e acupuntura, por exemplo, registradas pelo Ministério da Saúde como serviços de PIC. Em 2017 (de jan. a set.), verificou-se a oferta de 8.575 serviços $^{(8)}$.

Apesar da PNPIC priorizar a inserção das PIC na APS, os profissionais da Estratégia Saúde da Família (ESF) são os seus maiores promotores no SUS. De maneira geral, a formação de recursos humanos para o exercício de PIC, no Brasil, é considerada escassa e difusa, com limitações tanto na oferta quanto na qualidade do ensino profissional, sendo um dos maiores desafios para o aumento das PIC no Sistema Único de Saúde ${ }^{(9)}$. Estudo de 2011 admitiu que menos de $10 \%$ dos cursos de Medicina oferecidos no Brasil continham em seus currículos conteúdos de PICS ${ }^{(9)}$. 
O crescente aumento da população e da expectativa de vida, e a propagação de técnicas e novas especialidades médicas, que se multiplicam de modo assustador, são, além disso, a prosperidade e o drama maior do setor saúde, especialmente por atingirem as relações comunicativas, a ação coordenada dos novos conhecimentos e a integração da quantidade imensa de informações que se produz ${ }^{(10)}$.

O Brasil adotou a APS como política pública estruturada pela Atenção Básica à Saúde (ABS). De acordo com as diretrizes da PNPIC, esse espaço deve ser utilizado para a inclusão dessas práticas ${ }^{(11)}$. As PIC são importantes para os serviços de saúde. Além do cuidado completo, proporcionam benefícios como a visão holística do processo saúde-doença, o respeito pelas práticas de cuidado desenvolvidas por grupos e populações, e a promoção da qualidade de vida dos usuários ${ }^{(12)}$.

Destaca-se que, embora essa proposta terapêutica esteja mais voltada para grupos que vivem em condição social vulnerável, em termos de sua saúde mental e autonomia individual e comunitária, essa pesquisa tem o ensejo de mostrar que as PIC podem ser aplicadas em qualquer grupo de pessoas pertencentes as mais diferentes classes sociais, idades, situação socioeconômica e profissional, visto ser uma temática que valoriza o modo alternativo de cuidar das pessoas, respeitando a história de vida dos participantes, tendo como base de sustentação a construção de vínculos solidários e promoção da vida.

Ressalta-se a importância da subjetividade nas práticas e nos cuidados médicos e que a pluralidade disciplinar é o caminho para uma visão mais extensa e global do ser humano(11). E é nessa exposição que o repertório das PIC, com seu vasto conjunto de recursos, pode colaborar para a evolução da saúde pública, pois procede de uma tradição milenar de uso continuado e praticamente inalterado dos mesmos recursos tecnológicos pautados por natureza interdisciplinar.

Outro aspecto importante acerca das PIC é que, quando agregadas à APS, trazem relevantes contribuições para a humanização do cuidado e do atendimento ao usuário. Tal cuidado possibilita ao indivíduo que desempenhe maior autonomia sobre sua própria saúde, o que favorece a promoção do autocuidado e o cuidado da coletividade, além de ser criado um vínculo com os profissionais de saúde, tudo isso proporcionado pela utilização das PIC(13). Dessa forma, as práticas de promoção da saúde tendem a romper a demasiada fragmentação do processo saúdedoença, fortalecendo as articulações intersetoriais ${ }^{(14)}$, sendo, portanto, os usuários os personagens principais de seu processo de produção de saúde e da integralidade do atendimento.

O presente estudo permitiu aos autores a experiência com as PIC thetahealing ${ }^{(15)}$, hipnose ${ }^{(16)}$, quiropraxia e termalismo, e os terapeutas, que são profissionais de saúde do serviço a ser investigado, acolheram os mestrandos como participantes que integram o sistema de saúde, os quais respeitam os aspectos subjetivos e particulares do outro, promovendo o exercício da autonomia e o fortalecimento da vivência em PIC no campo da prática. Assim, diante desse contexto, o objetivo deste estudo foi relatar a vivência de mestrandos acerca do emprego das práticas integrativas e complementares como instrumento de construção de uma atenção integral à saúde em uma Unidade Básica de Saúde por profissionais de saúde do serviço.

\section{SÍNTESE DOS DADOS}

Trata-se de um relato de experiência de cunho descritivo, efetivado mediante a prática de campo de um grupo de mestrandos do Programa de Pós-Graduação em Enfermagem e Saúde (PPGES) da Universidade Estadual do Sudoeste da Bahia (UESB), Brasil. O local escolhido para realização da experiência foi uma Unidade de Saúde situada no Vale do Capão, distrito do município de Palmeiras, Chapada Diamantina, Bahia, entre os dias 21 a 25 de novembro de 2018, com a finalidade de vivenciar a realidade de uma unidade de saúde que implementou a PNPIC.

O cenário da pesquisa no campo da aprendizagem partiu das aulas teóricas e práticas da disciplina Política, Planejamento e Gestão, e a escolha do local se deu por ser referência no estado da Bahia na oferta das PIC no serviço de saúde. Na inserção da prática de campo, observou-se o planejamento e a gestão da referida política no serviço de saúde público.

Durante a visita técnica à unidade de saúde, percebeu-se que o atendimento da equipe de profissionais de saúde que atendiam aos programas da Atenção Básica $(A B)$ diferia do atendimento das outras unidades de saúde, pois os profissionais de saúde, capacitados na perspectiva das PIC, atendiam permitindo ao paciente escolher 0 tratamento a ser utilizado, fortalecendo a autonomia dos usuários do serviço.

A vivência dos mestrandos com as PIC foi iniciada com a técnica de thetahealing, seguidas de hipnose, quiropraxia e termalismo, realizadas por terapeutas da unidade, começando pela demonstração da técnica individual e, posteriormente, da técnica de forma coletiva, com os presentes na UBS. 
O thetahealing é uma prática de cura energética que ensina a identificar e liberar crenças e padrões que impedem alguém de ser feliz, tornando possível o aprendizado de olhar para si em busca da reconexão consigo mesmo e com sua essência ${ }^{(10)}$. Assim, a utilização dessa forma de tratamento tem como intuito a associação de outro tratamento ou a vinculação com o modelo normatizado, na tentativa de diminuir os custos, estimular medidas para prevenção de agravos e manutenção da saúde, por meio de tecnologias eficientes e seguras, as quais ressaltam a escuta acolhedora, a formação de vínculo com o profissional terapêutico e a conexão saudável do indivíduo com o meio ambiente e a sociedade, o que está de acordo com a PNPIC(17).

Na hipnose, o profissional abordou definições e mitos e, em seguida, realizou uma vivência grupal com todas as pessoas que se faziam presentes na sala de reunião da unidade. Logo após, realizou a técnica de forma individual, sendo escolhidos voluntários para demonstração da hipnose. A hipnose é situada como uma ferramenta de controle da dor, algo a ser manuseado por alguém externo à pessoa (o profissional de saúde) que representa um saber especializado, científico, enquanto processo psicoterápico, torna-se uma forma de ajudar o sujeito a lidar com o modo mais profundo da reconciliação consigo mesmo, que envolve a sua identidade e seu nicho afetivo de relações ${ }^{(11)}$.

Outras potencialidades das práticas vivenciadas podem ser mencionadas. O progresso da participação do usuário é um importante elemento a ser ressaltado, visto que se trata de um eixo central da promoção de saúde. No momento em que esse cenário ocorria, havia um terapeuta de quiropraxia, o qual exerce a função há vinte anos no município, que explicou o significado da quiropraxia e o objetivo do procedimento. Essa forma de terapia consiste na utilização das mãos humanas para amenizar dores e tensões, sendo um dos mais antigos recursos humanos para alívio de incômodos físicos. Com base nisso, sua forma originária tem por base o uso do procedimento fisioterapêutico, incluindo processos como avaliação, diagnóstico e modulação da força e ritmo dos movimentos manuais ${ }^{(18)}$.

Logo após sua explicação teórica sobre o significado e função de sua forma de terapia, o profissional fez a demonstração prática da técnica. Foi feita uma anamnese sobre origem, tempo e causa das dores apontadas por um voluntário, ao que foi possível verificar que a quiropraxia proporciona, na maioria das vezes, uma melhora imediata dos sinais e sintomas dos quais os pacientes reclamam. Assim, utilizando tal prática alternativa, há a possibilidade da abdicação da terapia alopática ou diminuir seu uso de forma considerável(18).

Ainda no Vale do Capão, às margens de uma cachoeira, foi oferecida aos presentes uma palestra ministrada pelo profissional do termalismo. Nesse momento, foi realizado um paralelo entre o conhecimento científico utilizado nas universidades e pós-graduações acerca das PIC e os limites encontrados pela ciência para legitimar tais práticas. Na sequência, as águas termais do Vale do Capão foram utilizadas para fins de finalização desse momento.

O termalismo consiste na utilização da água termal oriunda de fontes naturais ou artificialmente captadas para efeito terapêutico ${ }^{(19)}$. Enquanto prática de saúde e bem-estar, o recurso aos cuidados termais tem sua origem em tempos passados. Dessa forma, tocar nesse assunto nos dias atuais traz à tona a qualidade das águas naturais termais e os respectivos cuidados com a saúde.

É preciso mencionar, ainda, que a originalidade das contribuições das práticas analisadas à promoção da saúde consistem no trabalho com a percepção da unicidade do corpo como lugar da existência humana singularizada, em permanente relação perceptiva e plural com seus contextos de vida ${ }^{(20)}$.

Dessa forma, cabe afirmar que agregar as PIC à APS seja de grande importância ao considerar que elas trazem contribuições significativas no que tange à humanização do atendimento e do cuidado, o que viabiliza o resgate da autonomia dos pacientes sobre sua própria saúde. Além disso, o cuidado humanizado promove estratégias para o autocuidado e o cuidado da coletividade, uma vez que os usuários passam a ser os principais responsáveis pelo processo de produção de saúde e integralidade do atendimento(21).

Por outro lado, o número crescente das PIC tem se tornado um dos maiores desafios para a formação acadêmica e profissional, revelando a necessidade das universidades inserirem disciplinas referentes a essas práticas nos currículos $^{(22)}$. Além disso, é necessário considerar que a vivência de experiências com essas práticas auxilia para uma melhor formação profissional ao agregar conhecimento e possibilitar maior adesão a essas práticas.

O uso das práticas como thetahealing, hipnose, quiropraxia e termalismo permitiram expandir o olhar acerca do cuidado em saúde pessoal e comunitário. As vivências serviram para comprovar os benefícios, aperfeiçoar o conhecimento para as atividades no ensino sobre as PIC, sendo, de fato, uma experiência lisonjeira e importante, visto que forneceu conhecimento na prática, antes só vista na teoria ${ }^{(23)}$.

Trazer as PIC para a vivência dos mestrandos foi desafiador, transformador e recompensante. As compreensões de aprendizagem dos estudantes de pósgraduação poderiam levá-los a adequar-se a diferentes motivações e estratégias de aprendizagem, visto que poderiam melhorar os programas didáticos e o contexto educacional para obter um desempenho acadêmico mais apropriado ${ }^{(24)}$. 
A divulgação dessa experiência pode contribuir para que os gestores da área de saúde reconheçam a importância das PIC como instrumento de mobilização dos recursos pessoais e culturais na construção de redes de apoio social para promoção da saúde em consonância com os princípios do SUS ${ }^{(4)}$.

Espera-se que novos estudos sejam realizados, a fim de que se possa avaliar o impacto das PIC na promoção da saúde coletiva e seja realmente uma estratégia alternativa para potencializar a assistência prestada à comunidade. Como consequência dessa vivência, os mestrandos compartilharam o pensamento acerca da necessidade de promover experiências similares em outros ambientes de saúde, com o objetivo de propagar o conhecimento acerca das políticas públicas com os usuários, profissionais e a comunidade acadêmica.

A limitação deste estudo diz respeito ao tempo disponível para apresentação e desenvolvimento das PIC no curso de pós-graduação (mestrado), pois foi instigante conhecer as PIC com suas vinte e nove práticas em um curto período, solicitando que a disciplina Políticas, Planejamento e Gestão em Saúde tenha um tempo maior para a discussão teórica da temática. Nesse âmbito, consegue-se enxergar a necessidade da ampliação do tempo disponível para discutir, praticar e vivenciar as PIC, visto que é comum a formação de profissionais na área da saúde com parcial/total desconhecimento das PIC, o que configura dificuldade para a implantação nos serviços de saúde.

\section{CONCLUSÃO}

A experiência levou os participantes a refletirem que a utilização das PIC se apresenta como uma ótima possibilidade para auxiliar no pensar e no modo de fazer saúde a partir de um olhar sobre o sujeito e coletividade.

Dessa forma, a experiência foi considerada de suma importância para a formação profissional dos mestrandos em questão, vez que, como futuros profissionais da saúde e da docência, tais vivências proporcionam a expansão do conhecimento e da percepção acerca da validade e necessidade de agregar as PIC no campo do SUS.

\section{CONFLITOS DE INTERESSE}

Os autores declaram a inexistência de conflitos de interesses durante a realização do estudo.

\section{CONTRIBUIÇÕES}

Ivana Santos Ferraz e Ismar Eduardo Martins Filho contribuíram com a elaboração e delineamento do estudo. Alba Benemérita Alves Vilela e Eduardo Nagib Boery contribuíram com a redação e/ou revisão do manuscrito. Sérgio Donha Yarid contribuiu com a aquisição, análise e interpretação dos dados.

\section{REFERÊNCIAS}

1. Asher GN, Gerkin, J, Gaynes BN. Complementary therapies for mental health disorders. Med Clin North Am [Internet]. 2017 [acesso em 2020 Abr 29];101(5):847-64. doi: 10.1016/j.mcna.2017.04.004_

2. Tesser CD, Sousa IMC, Nascimento MC. Práticas integrativas e complementares na atenção primária à saúde brasileira. Saúde Debate [Internet]. 2018 [acesso em 2020 Abr 29]:42(spe1):174-88. doi: 10.1590/0103-11042018S112

3. Fischborn AF, Machado J, Fagundes NC, Pereira NM. A política das práticas integrativas e complementares do SUS: o relato da implementação em uma unidade de ensino e serviço de saúde. Cinergis [Internet]. 2016 [acesso em 2020 Abr 29];17. Disponível: https://online.unisc.br/seer/index.php/cinergis/article/view/8149

4. Brasil. Ministério da Saúde, Secretaria de Atenção à Saúde, Departamento de Atenção Básica. Portaria ${ }^{\circ}$ 971. Política Nacional de Práticas Integrativas e Complementares (PNPIC) no Sistema Único de Saúde. Diário Oficial da União, Brasília, 2006.

5. Brasil. Ministério da Saúde, Secretaria de Atenção à Saúde, Departamento de Atenção Básica. Portaria $\mathrm{n}$. 1600. Política Nacional de Práticas Integrativas e Complementares (PNPIC) no Sistema Único de Saúde. Diário Oficial da União, Brasília, 2017.

6. Ministério da Saúde (BR), Departamento de Atenção Básica. Ampliação da PNPIC [Internet]. 2017 [acesso em 2017 Dez 3]. Disponível em: http://189.28.128.100/dab/docs/portaldab/documentos/informe_pics_ maio2017.pdf 
7. Ministério da Saúde (BR). Portaria n. 971, de 3 de maio de 2006. Aprova a Política Nacional de Práticas Integrativas e Complementares (PNPIC) no Sistema Único de Saúde (SUS). Brasília: Ministério da Saúde; 2006.

8. Brasil. Ministério da Saúde, Secretaria de Atenção à Saúde, Departamento de Atenção Básica. Portaria $n^{\circ}$ 702. Política Nacional de Práticas Integrativas e Complementares (PNPIC) no Sistema Único de Saúde. Diário Oficial da União, Brasília, 2018.

9. Azevedo E, Pelicioni MCF. Práticas integrativas e complementares de desafios para a educação. Trab Educ Saúde. 2012; 9(3):361-78.

10. Telesi JE. Práticas integrativas e complementares em saúde, uma nova eficácia para o SUS. Estud Av [Internet]. 2016 [acesso em 2020 Abr 29];30(86):99-112. doi: 10.1590/S0103-40142016.00100007

11. Sousa IMC, Tesser CD. Medicina tradicional e complementar no Brasil: inserção no Sistema Único de Saúde e integração com a atenção primária. Cad Saúde Pública. 2017;33(1):00150215.

12. Ministério da Saúde (BR), Secretaria de Atenção à Saúde, Departamento de Atenção Básica. Política Nacional de Práticas Integrativas e Complementares no SUS: atitude de ampliação do acesso [Internet]. Brasília: Ministério da Saúde; 2015 [acesso em 2020 Jun 25]. Disponível em: http://bvsms.saude.gov.br/bvs/ publicacoes/politica_nacional_praticas_integrativas_compleme ntares_2ed.pdf

13. Paul P. Saúde e transdisciplinaridade. São Paulo: Edusp; 2013.

14. Ministério da Saúde (BR). Portaria $n^{\circ} 687$, de 30 de março de 2006. Aprova a Política Nacional de Promoção da Saúde (PNPS). Brasília: Ministério da Saúde; 2006.

15. Hinterberger T, de Von Haugwitz A, Schmidt S. Does a healing procedure referring to theta rhythms also generate theta rhythms in the brain? J Altern Complement Med [Internet]. 2016 [acesso em 2019 Fev 14];22(1):66-74. doi: 10.1089/acm.2014.0394

16. Neubern MS. Hipnose clínica e dores crônicas: rumo a uma perspectiva complexa. Psicol Estud [Internet]. 2016 [acesso em 2020 Jun 26];21(2):303-12. Disponível em: http://periodicos.uem.br/ojs/index.php/ PsicolEstud/article/view/30820

17. Schveitzer MC, Esper MV, Silva MJP. Práticas integrativas e complementares na atenção primária em saúde: em busca da humanização do cuidado. Mundo Saúde [Internet]. 2012 [acesso em 2019 Fev 14];36(3). Disponível: http://bvsms.saude.gov.br/bvs/artigos/mundo_saude/praticas_integrativas_complementares_ atencao_primaria.pdf

18. Lima KMSV, Silva KL, Tesser CD. Práticas integrativas e complementares e relação com promoção da saúde: experiência de um serviço municipal de saúde. Interface Com Saúde Educ [Internet]. 2014 [acesso em 2019 Mar 7];18(49):261-72. Disponível: http://www.scielo.br/scielo.php?pid=s1414$32832014000200261 \&$ script=sci_abstract\&tIng=pt_

19. Leandro ME, Leandro ASS. Da saúde e bem-estar/mal-estar ao termalismo. Sociologia [Internet]. 2015 [acesso em 2019 Mar 07];30:75-96. Disponível: http://www.scielo.mec.pt/scielo.php?script=sci_ abstract\&pid=S0872-34192015000300005\&lng=pt\&nrm=iso\&tlng=es

20. Galvanese ATC, Barros NF, Oliveira AFPL. Contribuições e desafios das práticas corporais e meditativas à promoção da saúde na rede pública de atenção primária do Município de São Paulo, Brasil. Cad Saúde Pública [Internet]. 2017 [acesso em 2019 Mar 07];33(12):e00122016. Disponível: http://www.scielo.br/scielo. php?pid=S0102-311X2017001205001\&script=sci_abstract\&tIng=pt_

21. Matos PC, Laverde CR, Martins PG, Souza JM, Oliveira NF, Pilger C. Práticas integrativas complementares na atenção primária à saúde. Cogitare Enferm [Internet]. 2018 [acesso em 2019 Mar 07];2(23):e54781. Disponível: http://www.scielo.br/scielo.php?script=sci_abstract\&pid=S0103-11042018000500174\&lng=en\&nr $\mathrm{m}=$ iso\&tlng=pt

22. Nascimento MC, Romano VF, Chazan ACS. Quaresma CH. Formação em práticas integrativas e complementares em saúde: desafios para as universidades públicas. Trab Educ Saúde [Internet]. 2018 [acesso em 2019 Mar 07];16(2):751-72. Disponível: http://www.scielo.br/scielo.php?script=sci_ abstract\&pid=S1981-77462018000200751\&lng=pt\&nrm=iso 
23. Climaco LCC, Ferraz IS, Almeida JS, Aragão SA, Reis LA, Martins IE Filho. A formação do discente de pósgraduação para a docência em práticas integrativas e complementares. Inter J Development Res [Internet]. 2019 [acesso em 2020 Mar 07];9(4):27276-80. Disponível: https://www.journalijdr.com/aformação-dodiscente-de-pós-graduação-para-docência-em-práticas-integrativas-e-complementares_

24. Campos F, Sola M, Santisteban-Espejo A, Ruyffelaert A, Campos-Sánchez A, Garzón I, et al. Concepções de fatores de aprendizagem em alunos de mestrado em ciências da saúde: um estudo comparativo com estudantes de ciências não-saudáveis e entre gêneros. BMC Med Educ [Internet]. 2018 [acesso em 2020 Maio 11];18(1). Disponível: https://www.researchgate.net/publication/325640304_Conceptions_of_learning_ factors_in_postgraduate_health_sciences_master_students_A_comparative_study_with_non-health_ science_students_and_between_genders

\section{Endereço para correspondência:}

Ivana Santos Ferraz

Universidade Estadual do Sudoeste da Bahia

Rua José Moreira Sobrinho, s/n

Bairro: Centro

CEP: 45.208-091 - Jequié - BA - Brasil

E-mail: ivanaferraz25@gmail.com

Como citar: Ferraz IS, Yarid SD, Vilela ABA, Boery EN, Martins IE Filho. Práticas integrativas e complementares na Atenção Primária à Saúde: relato de experiência. Rev Bras Promoç Saúde. 2020;33:10866. 